\title{
Dicer1 downregulation by multiple myeloma cells promotes the senescence and tumor-supporting capacity and decreases the differentiation potential of mesenchymal stem cells
}

\author{
Juan Guo', Youshan Zhao', Chengming Fei ${ }^{1}$, Sida Zhao ${ }^{1}$, Qingqing Zheng ${ }^{1}$, Jiying Su', Dong Wu' ${ }^{1}$ Xiao Li ${ }^{1}$ and \\ Chunkang Chang ${ }^{1}$
}

\begin{abstract}
Bone marrow mesenchymal stem cells (BMMSCs) facilitate the growth of multiple myeloma (MM) cells, but the underlying mechanisms remain unclear. This study demonstrates that the senescence of MM-MSCs significantly increased, as evidenced by a decrease in proliferation and increase in the number of cells positive for senescenceassociated $\beta$-galactosidase activity. Senescent MM-MSCs displayed decreased differentiation potential and increased tumor-supporting capacity. Dicer1 knockdown in the MSCs of healthy controls promoted cellular senescence and tumor-supporting capacity, while decreasing the differentiation capacity. Dicer1 overexpression in MM-MSCs reversed the effects on differentiation and reduced cellular senescence. In addition, decreased expression of the microRNA-17 family was identified as a favorable element responsible for increasing senescence, with the expression of p21 increased in Dicer1 knockdown cells. Furthermore, we observed decreased expression of miR-93 and miR-20a in MMMSCs, while upregulation of miR-93/miR-20a decreased cellular senescence, as evidenced by the increased p21 expression. Importantly, we found that myeloma cells could induce the senescence of MSCs from healthy controls, as observed from the decreased expression of Dicer 1 and miR-93/miR-20a and increased expression of p21. Overall, MM cells downregulate Dicer1 in MSCs, which leads to senescence; in turn, senescent MSCs promote MM cell growth, which most likely contributes to disease progression.
\end{abstract}

\section{Introduction}

Multiple myeloma (MM) is a malignancy characterized by plasma cell proliferation, initially in the bone marrow microenvironment ${ }^{1}$. This tissue is a complicated network of extracellular matrix and various cells ${ }^{2}$. Undifferentiated and pluripotent bone marrow mesenchymal stem cells (BMMSCs) are the principle members of the bone

\footnotetext{
Correspondence: Chunkang Chang (changchunkang@sjtu.edu.cn)

'Department of Hematology, Shanghai Jiao Tong University Affiliated Sixth People's Hospital, 200233 Shanghai, China
}

Edited by $Y$. Wang marrow microenvironment. BMMSCs promote MM cell proliferation via different factors, leading to increased tumor supporting capacity and the development of drug resistance. Evidence has shown that MSCs isolated from MM (MM-MSCs) exhibit different behavior from that exhibited by the MSCs from healthy controls (HC-MSCs), including differential gene expression, impaired cytokine release, and decreased osteogenic differentiation poten$\mathrm{tial}^{3-6}$. In MM, the interaction between BMMSCs and MM cells plays a pivotal role in MM development. MM cells attach to BMMSCs and release cytokines that

\section{(c) The Author(s) 2018}

(c) (i) Open Access This article is licensed under a Creative Commons Attribution 4.0 International License, which permits use, sharing, adaptation, distribution and reproduction cc in any medium or format, as long as you give appropriate credit to the original author(s) and the source, provide a link to the Creative Commons license, and indicate if changes were made. The images or other third party material in this article are included in the article's Creative Commons license, unless indicated otherwise in a credit line to the material. If material is not included in the article's Creative Commons license and your intended use is not permitted by statutory regulation or exceeds the permitted use, you will need to obtain permission directly from the copyright holder. To view a copy of this license, visit http://creativecommons.org/licenses/by/4.0/. 


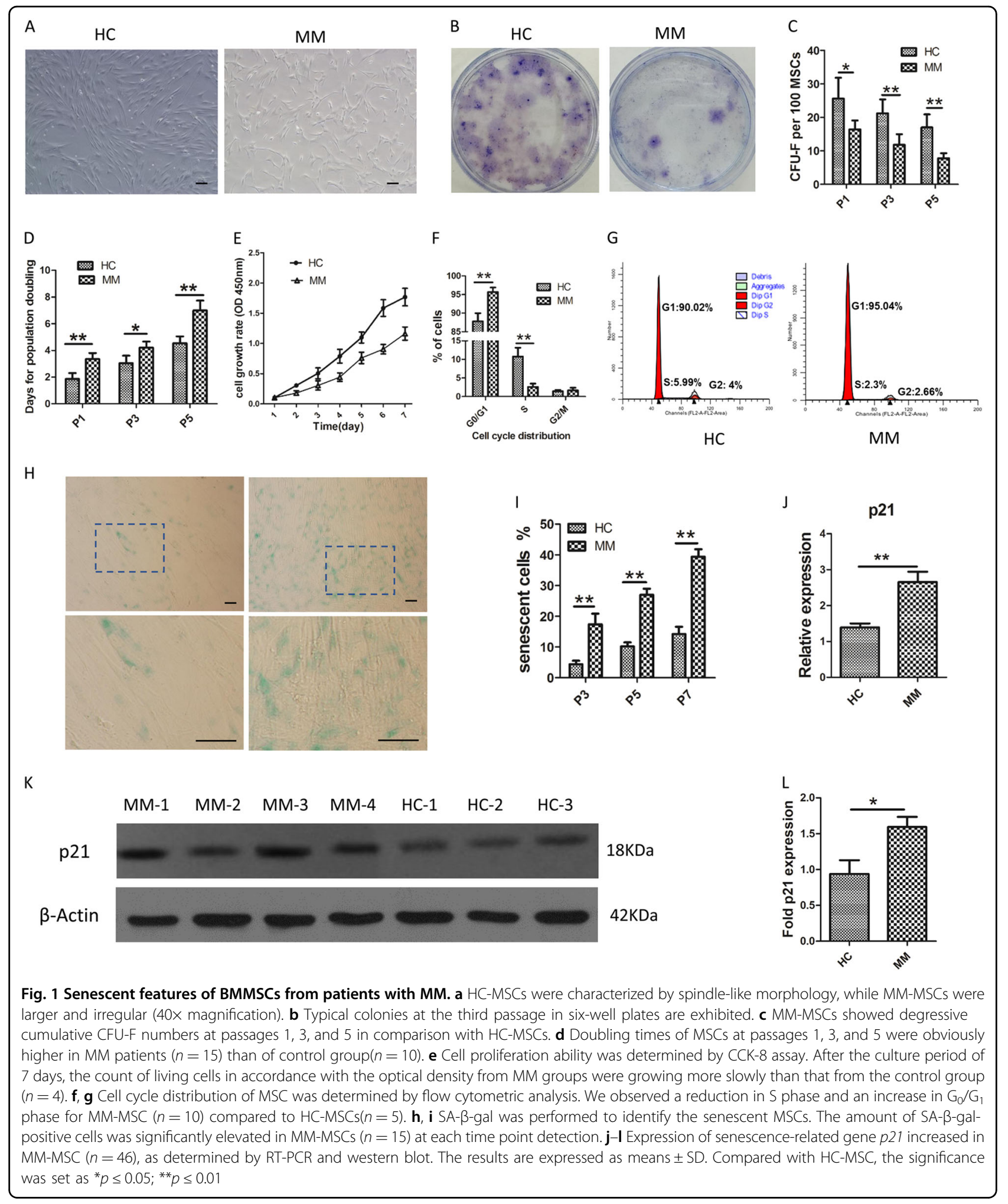

promote MM cell viability, migration, and invasive ability, ultimately enhancing MM progression ${ }^{7}$. This interaction usually leads to complicated changes, including dysregulation of mRNA and miRNA expression and activation or deactivation of signaling pathways, but the underlying mechanisms remain unclear. For example, the development of a senescence-like state in BMMSCs, and thereby a modulated secretory profile, has been reported to 
worsen osteogenic differentiation potential ${ }^{8-11}$. Senescence is a cellular state corresponding with a decrease in proliferation ability and variation in the release of proinflammatory cytokines and growth factors ${ }^{12}$. Senescent BMMSCs exhibit an increased senescence-associated $\beta$ galactosidase activity (SA- $\beta$-gal) and irregular cell morphology ${ }^{13,14}$. Generally, cell cycle of the senescent cells is often arrested at the $G_{0} / G_{1}$ phase, in combination with the upregulation of different cell cycle inhibitors such as $\mathrm{p} 21^{15-17}$. Increasing body of report suggests that microRNAs (miRNAs) take part in the regulation of MSC senescence $^{18,19}$. Furthermore, they play an important part in the self-renewal and differentiation of MSCs. As previously indicated, MM-MSCs show a different miRNA profile to that of $\mathrm{HC}$-MSCs, but the roles of these deregulated miRNAs in MM-MSCs are not clearly known $^{20,21}$. The loss of Dicer1, an RNAse III endonuclease essential for miRNA biogenesis, and the ablation of mature miRNA result in the inhibition of cell proliferation and the induction of a premature senescence phenotype $^{22-24}$. Our previous study showed that Dicer1 expression is reduced in MSCs from myelodysplastic syndrome patients (MDS) and that this down-regulation promotes cellular senescence and decreases the stem cellsupporting capacity of these cells ${ }^{17}$. However, the effect of the Dicer1 gene on the pathogenesis of MM has not been studied.

In this study, we studied the senescent features of BMMSCs derived from MM patients and explored the biological function of Dicer1 in the senescence of MMMSCs. Finally, we analyzed the decreased differentiation potential and the enhanced tumor-supporting capacity of MM-MSCs.

\section{Results}

Senescent features of BMMSCs from patients with MM

HC-MSCs were characterized by spindle-like morphology, while MM-MSCs were larger and irregular (Fig. 1a). The clonogenic ability of the MSCs were identified by colony forming unit fibroblast (CFU-F). MMMSCs showed degressive cumulative CFU-F numbers at passages 1,3 , and 5 in comparison with HC-MSCs $(p<$ 0.05 , Fig. 1b, c). In addition, the doubling times of MSCs at passages 1,3 , and 5 were obviously higher in MM patients than of control group (Fig. 1d). Cell proliferation ability was determined by CCK- 8 assay. After the culture period of 7 days, the count of living cells in accordance with the optical density from MM groups were growing more slowly than that from the control group (Fig. 1e). To demonstrate whether the reductive MM-MSCs growth was caused by weak proliferation or intensive apoptosis, the distributed population of cells in each cell cycle phase was evaluated. No Sub- $G_{0}$ peak, in accordance with hypodiploid apoptotic cells, was existed in the BMMSC cultures $(<1 \%)$. Nevertheless, the percentage of MMMSCs in $\mathrm{G}_{0} / \mathrm{G}_{1}$ phase was increased to $95.66 \pm 1.26 \%$ (vs $87.78 \pm 2.20 \%$; *** $p<0.01$ ), while the percentage of MMMSCs in S phase was reduced to $2.64 \pm 0.87 \%$ (vs $10.77 \pm$ $2.39 \%$; * $p<0.05$ ) when compared with that of HC-MSCs (Fig. 1f, g).

SA- $\beta$-gal was performed to identify the senescent MSCs. The number of SA- $\beta$-gal-positive cells was significantly elevated in MM-MSCs at each time point detection (Fig. 1h, i). From passage 3, the median percentage of senescent cells in HC-MSCs accounted for $4.4 \%$ of all HC-MSCs. At a cut off value of $4.4 \%$, increased senescence was observed in $86.67 \%(26 / 30)$ of MM-MSCs. Next, we measured the expression of $p 21$, which is involved in the regulation of cell senescence. Using RTPCR and western blotting, we found an increase in $p 21$ in MM-MSCs in comparison with the control group (Fig. 1j-l). Moreover, the level of $p 21$ expression increased in MM-MSCs (S-MM-MSCs, SA- $\beta$-gal-positive cells $\geq 4.4 \%$ ) when compared with non-senescent MM-MSCs (NS-MM-MSCs, SA- $\beta$-gal-positive cells $<4.4 \%$ ).

In accordance with the above phenomena, primary MSCs $\left(\mathrm{CD} 271^{+}\right)$from MM patients also exhibited raised cell senescence, which was displayed by an obviously increased amount of SA- $\beta$-gal positive cells and increased $p 21$ expression level, in comparison with the healthy control group. The collective data indicate that the proliferation capacity decreased and the senescence increased in MSCs from MM patients.

\section{Senescent MM-MSCs exhibited decreased differentiation}

On account of that cell dysfunction is relevant to cell senescence, we identified the ability of senescent MMMSC and HC-MSCs to differentiate and to promote tumor cell proliferation. The osteoblastic and adipogenic differentiation capabilities of MSCs were assessed by immunohistochemical method and associated genes expression analysis. In comparison with $\mathrm{HC}-\mathrm{MSCs}$ and NS-MM-MSCs, senescent MM-MSC showed significantly reduced osteogenic differentiation potential, which is indicated by the results of mineralization analysis and activated ALP evaluation (Fig. 2a-c). In accordance with the immunohistochemical staining analysis, the mRNA expressions of RUNX2 and $A L P$, which play crucial role in the process of osteoblastic differentiation, were notably decreased in senescent MM-MSCs undergoing osteoblastic differentiation (Fig. 2d, e). The ability of adipogenic differentiation was identified by Oil Red-O staining, and it was increased significantly in HC-MSCs than that in senescent MM-MSCs (*** $p<0.01$; Fig. 2f, g). The differences of the adipogenic differentiation capability between HC-MSCs and senescent MM-MSCs were also revealed in the expressions of FABP4 and C/EBPa mRNAs (Fig. 2h, i). 


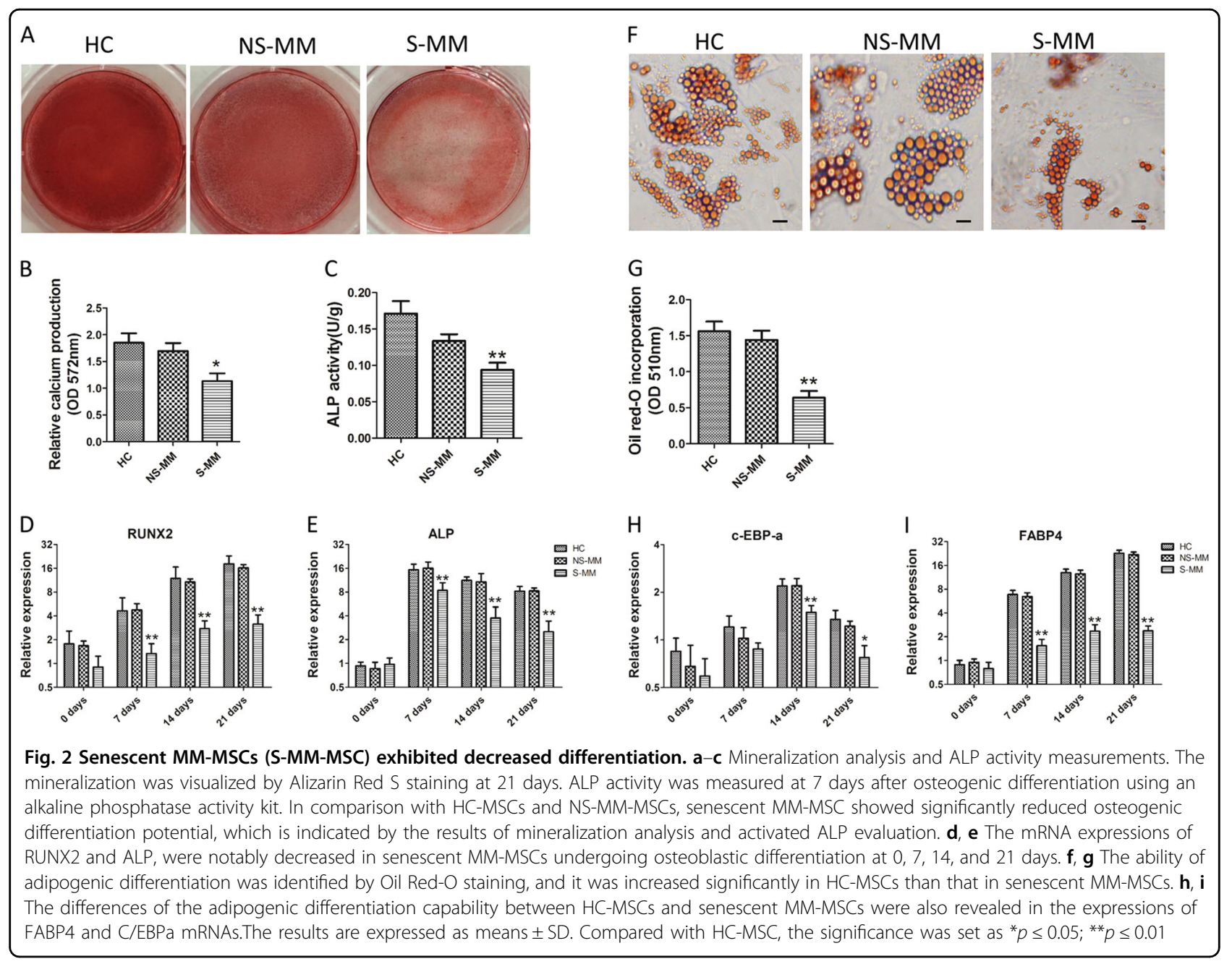

In addition, there exists a significant difference in these two group of MM patients. The patients with high $\beta$-gal staining MM-MSCs (S-MM) showed different disease progression compared to those with low $\beta$-gal staining MM-MSCs(NS-MM). Progression free-survival (PFS) was calculated from the date of the diagnosis to the date of progression. Patients with less than $4.4 \%$ SA- $\beta$-galpositive cells at diagnosis, had significantly longer PFS (mean of $29 \mathrm{v} 11$ months; $p=0.014$; Supplementary Figure S1) than patients with more than $4.4 \% \mathrm{SA}-\beta$-galpositive cells.

Low expression of Dicer1 was exhibited in MM-MSCs, and MM cells induced senescence in HC-MSCs

The attenuation of Dicer1 resulted in rapid cell senescence in a variety of cells. We examined the level of Dicer1 in in vitro expanded and primary $\left(\mathrm{CD} 271^{+}\right) \mathrm{MSCs}$ derived from MM patients and healthy controls. Results from RT-PCR and western blot exhibited that the expression of Dicer1 was declined in MM-MSCs (Fig. 3a-d).
To determine whether myeloma cells could affect MSCs, HC-MSCs were co-cultured with myeloma cell lines (such as NCI-H929, OPM-2, and KMS-12-BM) or with primary $\mathrm{CD} 138^{+}$tumors cells from three $\mathrm{MM}$ patients for $24 \mathrm{~h}$ and the expressions of Dicer $1, p 21$, miR93 and miR-20a was determined by RT-PCR. We used trans-well inserts with $1-\mu \mathrm{m}$ pores. MM cell lines or MM primary cells were cultured in the upper chamber of the inserts. We observed that myeloma cells could significantly decrease the expression of Dicer1, $p 21$, miR-93 and miR-20a in HC-MSCs (Fig. 3e, f). The cells in the lower chamber were stained by SA- $\beta$-gal assay and results confirm that the cells were undergoing senescence. A few of staining were identified in the control MSC, while SA$\beta$-gal activity was clearly obvious in the co-cultured MSC (data not shown).

\section{Knockdown of Dicer1 induced senescence and inhibited differentiation of HC-MSCs}

We then constructed a recombinant lentivirus to decrease the expression of Dicer1 to further study the biological effect 


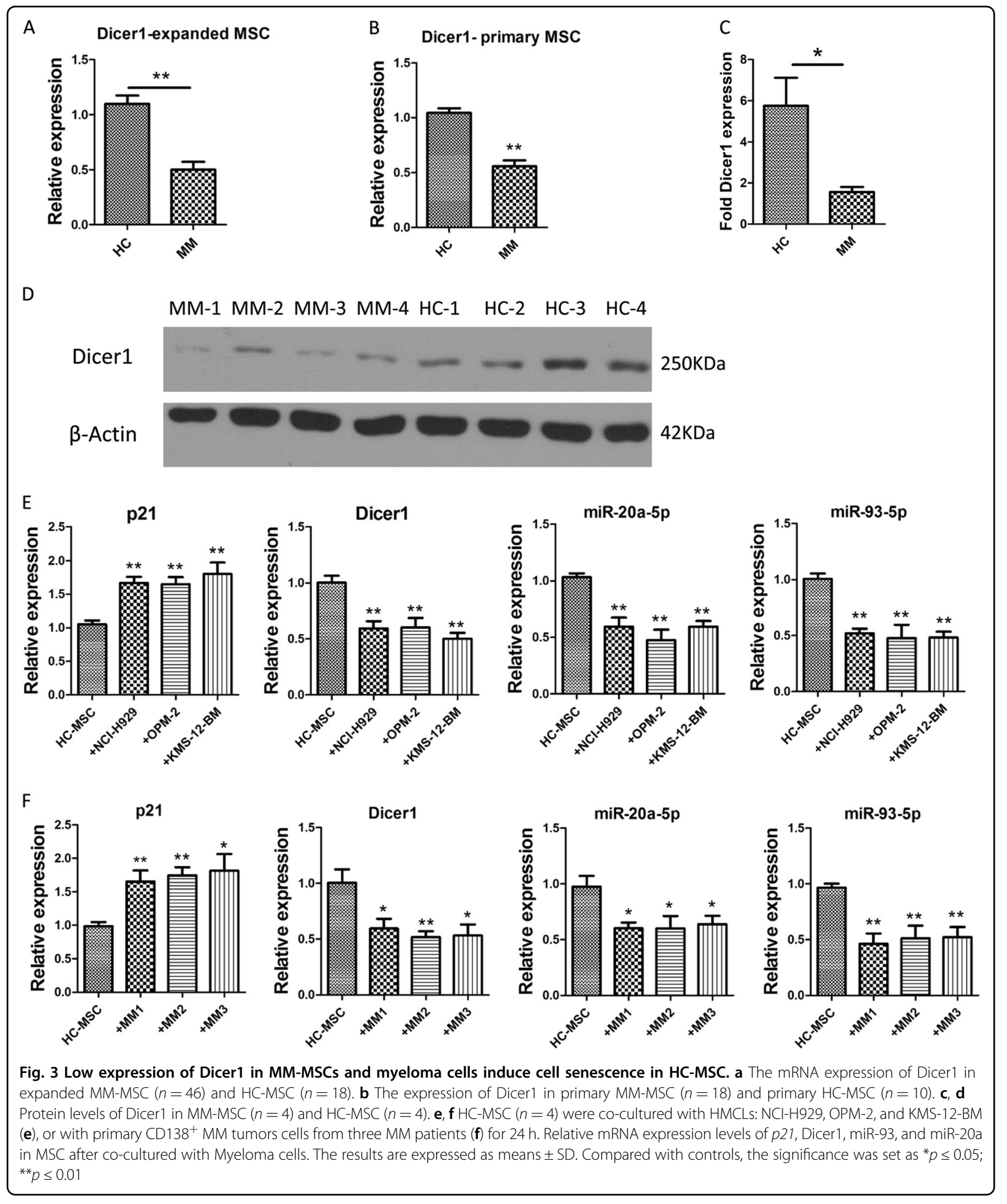

of Dicer1 on MSC senescence. It is displayed in Fig. 4a-d that the expressions of Dicer1 mRNA and protein were dramatically decreased compared to that of the control group. The proliferation of MSCs treated with Dicer1 knockdown (KD) was obviously inhibited in comparison with either control MSCs or the negative group (Fig. 4g).

To demonstrate whether the cells were growing senescent, Dicer1-KD MSCs or control groups were identified 


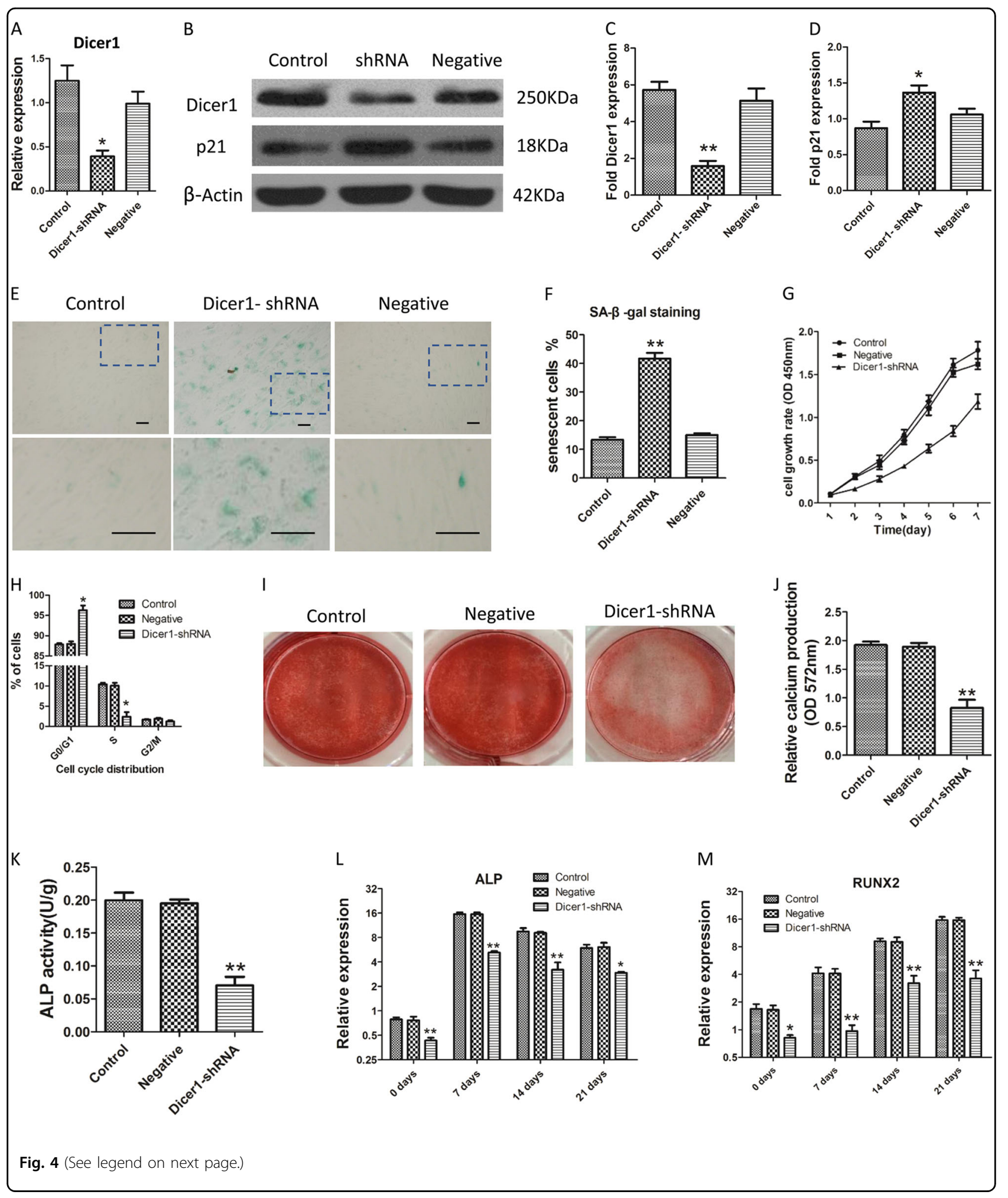

once they reached confluence. On the 14th day, Dicer1KD MSCs stopped division and exhibited a large, flattened shape. Cells were collected and replanted in fresh medium at higher or lower densities and the proliferation cannot be promoted. To identify that Dicer1-KD MSCs were becoming senescence, cells were then stained by SA- $\beta$-gal 
Fig. 4 Knockdown of Dicer1 induced senescence and inhibited osteogenic differentiation of HC-MSCs. a-d The expressions of Dicer1 mRNA and protein were dramatically decreased compared to that of the control group. And the protein expression of p21 increased. e Representative micrographs after SA- $\beta$-gal staining of Dicer1-KD MSC (shRNA), negative MSC (transfected with control lentiviruses) and control-MSC (HC-MSC without transfection) (100x magnification). f One hundred MSC per sample were counted using light microscopy, and the percentages of SA- $\beta$-galpositive cells were determined. The average of three replicates is displayed. $\mathbf{g}$ The proliferation of MSCs treated with Dicer1 knockdown (KD) was obviously inhibited in comparison with either control MSCs or the negative group. $\mathbf{h}$ Cell cycle analysis of Dicer1-KD MSC by flow cytometric analysis. Dicer $1 \mathrm{KD}$ caused an increasing proportion of cells in the G1 phase and a decrease of those in the S phase without inducing apoptosis. $\mathbf{i}$ After 21 days of osteogenic induction, Alizarin red S staining was performed to visualize osteogenic differentiation. Representative original images of BMMSCS derived from control-MSC (HC-MSC without transfection), negative MSC (transfected with control lentiviruses), Dicer1-KD MSC are shown. $\mathbf{j}$ Relative calcium production (OD $572 \mathrm{~nm}$ ) by Dicer1-KD MSC, was significantly lower after 21 days of differentiation as compared with controls. $\mathbf{k}$ The ALP activity of Dicer1-KD MSC was significantly lower than that of controls after 3 days culturing in osteogenic medium (OM). I, $\mathbf{m}$ Relative RUNX2 and ALP mRNA expression levels. The average of three replicates is displayed. Compared with controls, the significance was set as ${ }^{*} p \leq 0.05$; ** $p \leq 0.01$

assay. Light staining was determined in the control and negative groups, while SA- $\beta$-gal activity was quite clear in the Dicer1-KD MSCs (Fig. 4e). Exceeding 40\% of the Dicer1-KD MSCs were growing senescence after 7 days, in comparison with less than $14 \%$ of the control and negative groups (Fig. 4f). The above results reveal that down-regulation of Dicer1 is effective for MSC senescence induction. Furthermore, Dicer1 KD caused an increasing proportion of cells in the $G_{1}$ phase and a decrease of those in the $\mathrm{S}$ phase without inducing apoptosis (Fig. 4h).

We measured the biological role of Dicer1-KD on the capability of MSCs to differentiate into osteogenic and adipogenic cells. Dicer1-KD MSCs were treated with osteogenic induction and exhibited decreased mineralization when stained with Alizarin red (Fig. 4i). Furthermore, they showed a reduction in ALP activity compared with negative MSCs and control cells $(* * p<$ 0.01) (Fig. 4j, k). In the process of osteogenic induction, mRNA levels of ALP and RUNX2 were elevated significantly in the control MSCs. On the contrary, the expressions of these mRNAs increased mildly in Dicer1KD MSCs (Fig. 4l, m).

We also assessed the ability of MSCs differentiating into an adipogenic lineage treated with knockdown of Dicer1. On Day 21, Oil red-O-stained cultures revealed that excessive $91 \%$ of control MSCs could differentiate into adipocytes, exhibiting plenty of cellular lipid droplets. On the contrary, the cellular lipid amount in Dicer1-KD MSCs was rare, consisting of small droplets (Supplementary Figure $\mathrm{S} 2 \mathrm{~A}$ ). In the adipogenic inductive process, the expressions of C/EBPa and FABP4 were significantly elevated. After 14 days, control MSCs showed an obvious raise in $\mathrm{C} / \mathrm{EBPa}$ and $\mathrm{FABP} 4$ in comparison with undifferentiated cells. On the contrary, Dicer1-KD MSC showed only a slight increase in mRNA expressions, with a 1.64-fold raise in C/EBPa and a 0.64-fold raise in FABP4 when in comparison with undifferentiated cells (Supplementary Figure S2B, C).

\section{Down-regulation of Dicer1 in MSCs stimulated the proliferation and reduced apoptosis of $\mathrm{MM}$ cells}

Dicer1 knockdown increased the tumor-supporting properties of MSCs. NCI-H929 cells were co-cultured with the following MSCs: control-MSCs (HC-MSCs without transfection), negative MSCs (transfected with control lentiviruses), Dicer1-KD MSCs (shRNA), and MM-MSCs for 24h. Cell cycle behavior of NCI-H929 cells was identified by Click-iT Edu Flow Cytometry Assay. Fluorescence-activated cell analysis plots from one representative experiment showing the cell cycle plots for monocultured MM cell lines (NCI-H929) or with MSCs as indicated. The location of cells in different phases of cell cycle is shown in the left histogram, and the percentage of cells in $\mathrm{S}$ is obtained for each condition (Fig. 5a). The results of the Edu assay showed that Dicer1KD MSCs and MM-MSC promoted myeloma cell proliferation, and that the proportion of $\mathrm{S}$ phase cells was increased compared with that in the HC-MSC or MSCNegative groups (Fig. 5a, b).

Relapse and chemo resistance are the main features of the clinical course of MM, and it is well-known that the bone marrow microenvironment protects MM cells against chemotherapy. Here we examined whether MMMSCs could protect MM cells against chemotherapyinduced apoptosis. Bortezomib is a clinically available proteasome inhibitor that is currently among the most potent chemotherapeutic drugs used in the treatment of MM. When the human MM cell line NCI-H929 or MMderived $\mathrm{CD} 138^{+}$cells were cultured in complete medium with $5 \mathrm{nM}$ Bortezomib for $48 \mathrm{~h}$, approximately $33.4 \%$ apoptotic cells (early and late) were observed. However, in the presence of MSC-Dicer1-shRNA and MM-MSC, the proportion of apoptotic cells significantly decreased to $9.8 \%$ and $10.1 \%$, respectively (Fig. $5 \mathrm{c}-\mathrm{e}$ ).

MSCs interact with MM cells, releasing cytokines which promote the viability of MM cells. Variational chemokine levels could cause the promoted tumor supporting effect of MM-MSCs. The expression of cytokines was then 


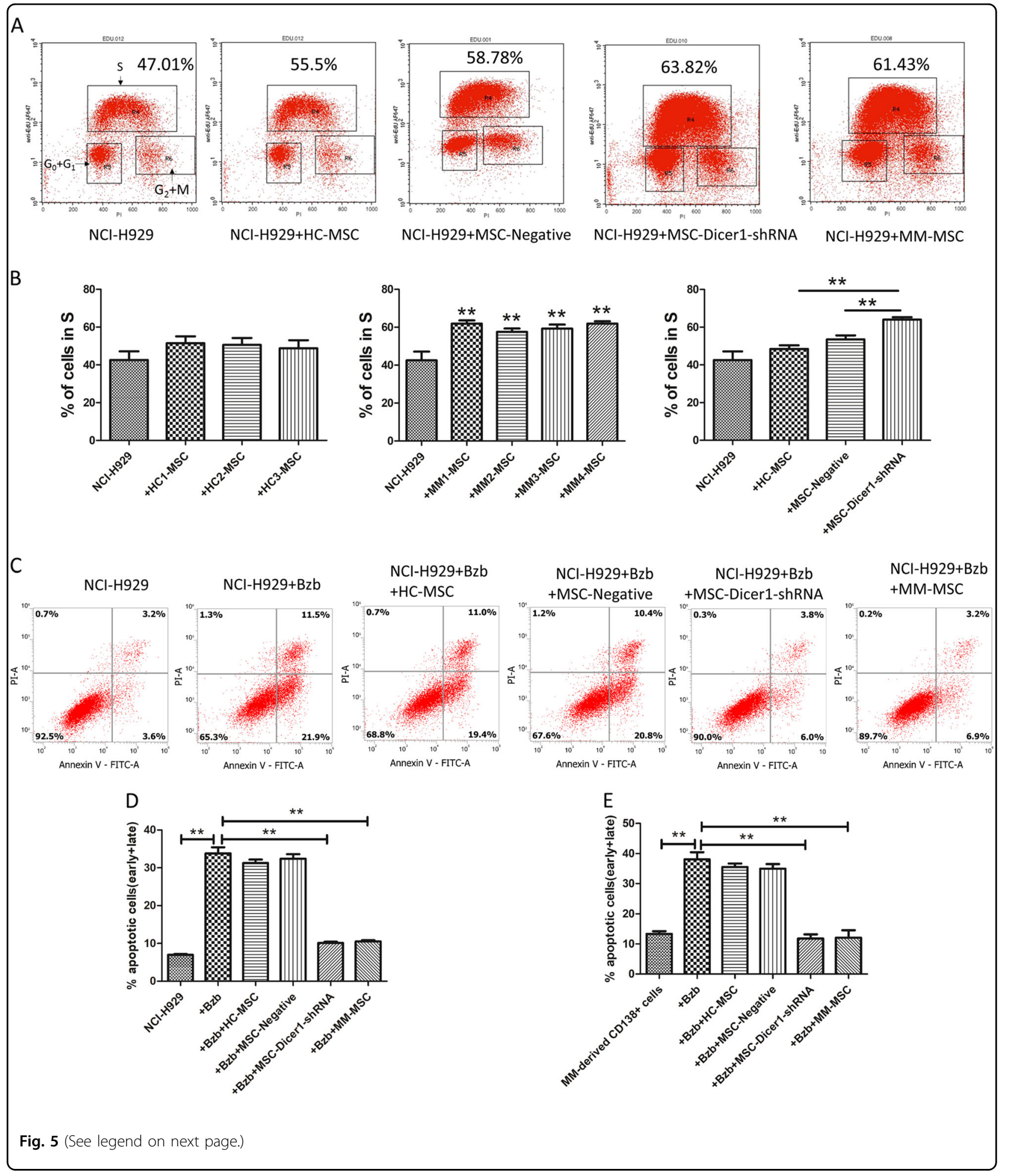

detected in MM-MSCs and they showed an increased expression of GDF-15 (growth differentiation factor-15), DKK-1 (the Inhibitor of the Wnt signaling pathway-Dickkopf1), and IL-6 (interleukin-6) compared with control
MSCs. However, the level of SDF-1(stromal cell derived factor 1) was significantly under-expressed in MM-MSCs compared to HC-MSCs. Cytokines were evaluated by realtime RT-PCR and ELISA. (Supplementary Figure S3). 


\begin{abstract}
Fig. 5 Down-regulation of Dicer1 in MSCs stimulated MM cell proliferation and protected MM cells against apoptosis in vitro. a NCl-H929 were co-cultured with MSCs: control-MSC (HC-MSC without transfection), negative MSC (transfected with control lentiviruses), Dicer1-KD MSC (shRNA), and MM-MSC for $24 \mathrm{~h}$. Cell cycle behavior of H929 was assessed by Click-iT Edu Flow Cytometry Assay. Fluorescence-activated cell analysis plots from one representative experiment showing the cell cycle plots for cultured alone MM cell lines(H929) or with MSCs as indicated. The location of cells in different phases of the cell cycle is indicated in the left histogram, and the percentage of cells in $\mathrm{S}$ is given for each condition. $\mathbf{b}$ Summary of results from separate experiments. H929 cultured alone (control), or co-cultured with HC-MSCs from three healthy controls(H929 + HC-1-3MSC), or co-cultured with MM-MSCs from four different patient samples (H929+ MM1-4-MSC), or co-cultured with negative MSC (transfected with control lentiviruses), Dicer1-KD MSC (shRNA) for $24 \mathrm{~h}$ were assessed for the proportion of cells in S by Click-iT Edu Flow Cytometry Assay. The results are expressed as means $\pm \mathrm{SD}$. The average of three replicates is displayed. Compared with controls, the significance was set as ${ }^{*} p \leq 0.05 ;{ }^{* *} p \leq 0.01$. c-e MSCs were cocultured in complete growth medium with MM cells (NCl-H929 or CD138 ${ }^{+}$MM cells) for $48 \mathrm{~h}$ at a ratio of 1:10 (MSCs/MM cells). As measured by AnnexinV/PI staining, MM-MSCs could protect against Bortezomib-induced apoptosis at a ratio of 1:10 (MSCs/MM cells). Similarly, MSCDicer1-shRNA can also protect Bortezomib-induced apoptosis of MM cells. The results are expressed as means \pm SD. The average of three replicates is displayed. Compared with controls, the significance was set as ${ }^{*} p \leq 0.05 ;{ }^{* *} p \leq 0.01$
\end{abstract}

Upregulation of Dicer1 reversed the senescent features of MM-MSCs and restored their differentiative capacity

In the following experiment, we performed MM-MSC transfected with an adenovirus carrying the Dicer1 gene (AD-Dicer1) or green fluorescent protein (AD-GFP). The mRNA and protein expressions of Dicer1 was significantly elevated in AD-Dicer1 MSCs (Fig. 6a-d) levels. It was shown that MM-MSCs transfected with AD-Dicer1 proliferate more quickly than either MM-MSCs or MMMSCs transfected with adenovirus carrying green fluorescent protein (AD-GFP) (Fig. 6h). There were no evident SA- $\beta$-gal-positive cells in MM-MSCs with Dicer1 overexpression (Fig. 6e, f). Furthermore, overexpression of Dicer1 gave rise to an increasing number of cells in the $\mathrm{S}$ phase and decreased in the G1 phase (Fig. 6g). Furthermore, AD-Dicer1 MSCs showed increasing osteoblastic (Fig. 6i-m) and adipogenic differentiation ability (Supplementary Figure S4) in comparison with MSCs which was treated with GFP vectors transfection.

\section{MiR-17 family members participated in Dicer1 KD-induced senescence}

To identify whether lower expression of Dicer1 in MSC could show effect on miRNA biogenesis and take part in cell senescence, we detected a number of specific miRNAs which possibly target senescence-associated molecules, such as $\mathrm{p} 21$. miRBase, PicTar and Targetscans were performed to screen targets. miR-20a expression was significantly decreased in Dicer1-KD MSCs in comparison with both control MSCs and the negative group (Fig. 7a, b). Decreased expressions of miR-93 and miR-20a was also appeared in MM-MSCs when compared with HCMSCs. To demonstrate that p21 is a target of miR-93 and miR-20a in MM-MSCs, studies involving miR-93/miR20a up-regulation were employed. MM-MSCs were transfected with miR-93 and miR-20a harboring lentiviruses, and $p 21$ levels after $48 \mathrm{~h}$ transfection was also detected. In three analyzed samples of MM-MSCs, the overexpression led to decreased $p 21$ expression (Fig. $7 \mathrm{~d}$ ) compared with that in cells transfected with scrambled control lentivirus.

\section{Discussion}

MM cells are believed to have a reciprocal interaction with the surrounding tumor microenvironment, which consists of endothelial cells, osteoclasts, fibroblasts, macrophages, and other cells. These cells play a crucial part in support of the proliferation, survival, chemo-resistance, and migration of MM cells ${ }^{5}$. A lot of studies showed that the role of the $\mathrm{BM}$ microenvironment in cytokines and growth factors secretion that could promote MM pathological mechanism and therefore take part in disease progression. After co-culture with MM cells, normal MSCs showed a phenotype as similar with that of MM$\mathrm{MSCs}^{25}$, demonstrating that MM cells induce this phenotypic change, either by immediate contact or by soluble elements $^{3,26}$. The current results indicated a new mechanism by which BMMSCs act as an oncogenic factor in MM.

In the present study, we first confirmed that MM-MSCs were more likely to be senescent than HC-MSCs. Although MSCs from all MM patients were expanded successfully in vitro, MM-MSCs showed a reduced expansion potential when compared to HC-MSCs. These observations are in line with the findings of several previously published studies, but conflict with other research in which MM-MSCs had normal proliferative characteristics. The typical features of cellular senescence include enlarged morphology, decreased cellular proliferation, and increased SA- $\beta$-gal expression. Exactly as expected, most of the cultured MM-MSCs were larger, flatter, irregular and expressed observably higher p21 (the senescence-related molecule). It is clear that the amount of SA- $\beta$-gal positive cells was significantly higher in MMMSCs than HC-MSCs. Our findings are in agreement with previous studies, but these studies did not detail the mechanisms and functional changes associated with senescence in the pathogenesis of MM. 


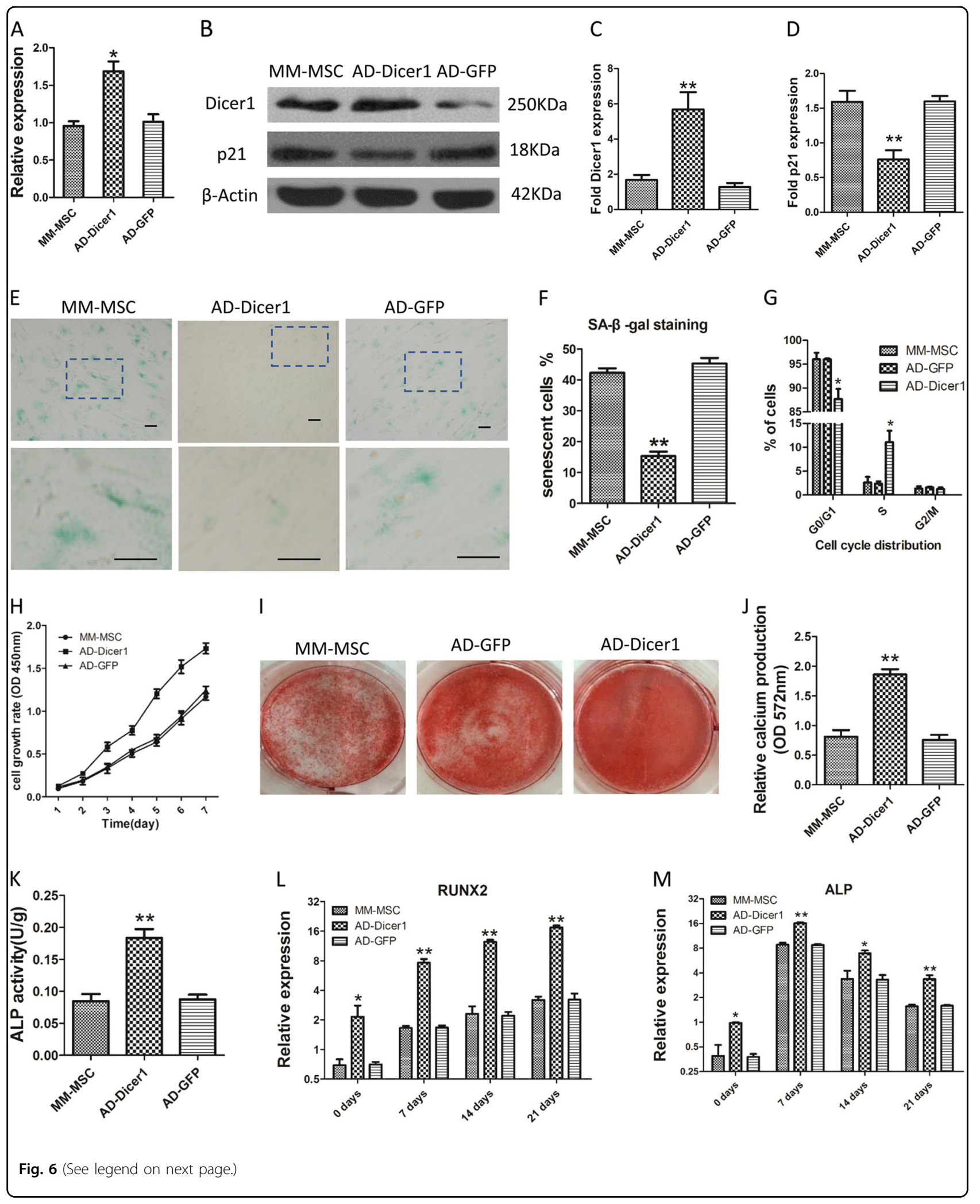


Fig. 6 Upregulation of Dicer1 reversed the senescent features and osteogenic differentiation of MM-MSCs. a-d The expressions of Dicer 1 mRNA and protein were dramatically increased after adenovirus transfection. And the protein expression of p21 decreased. e Representative micrographs after SA- $\beta$-gal staining of control MSC (MM-MSC without transfection), AD-Dicer1 MSC (MM-MSC transfected with Dicer1 adenovirus lentiviruses) and AD-GFP (MM-MSC transfected with control lentiviruses) (100x magnification). $\mathbf{f}$ The percentages of SA- $\beta$-gal-positive cells. $\mathbf{g}$ Cell cycle analysis of Dicer1-KD MSC by flow cytometric analysis. Dicer1 AD caused an increasing proportion of cells in the $\mathrm{S}$ phase and a decrease of those in the G1 phase. $\mathbf{h}$ MM-MSCs transfected with AD-Dicer1 proliferate more quickly than either MM-MSCs or MM-MSCs transfected with AD-GFP. i Typical images after Alizarin Red S staining on day 21 of osteogenic differentiation. $\mathbf{j}$ Relative calcium production (OD $572 \mathrm{~nm}$ ) by AD-Dicer1 MSC was significantly higher after 21 days of differentiation as compared with controls. $\mathbf{k}$ The ALP activity of AD-Dicer1 MSC was significantly increased after 3 days osteogenic differentiation. I, $\mathbf{m}$ Relative RUNX2 and ALP mRNA expression levels. The results are expressed as means \pm SD. The average of three replicates is displayed. Compared with controls, the significance was set as ${ }^{*} p \leq 0.05,{ }^{* *} p \leq 0.01$

The characteristics of senescent MSCs include changes in cellular functions. We demonstrated that senescent MM-MSCs displayed decreasing differentiation capability and increased tumor supporting ability. The depressed osteogenic and adipogenic potentials in senescent MMMSCs have been verified in vitro by quantitative and continuous detection. In particular, some cell surface molecules and subsequent downstream signaling pathways take part in the regulation of MM-related bone destroying process, in which the balance of bone resorption and formation are no longer acts as a result of the increasing activity of osteoclasts, but rather the osteoblast activity is decreased, leading to an uncoupled or severely imbalanced bone remodeling process. In addition, our previous study showed that MM patients exhibit an impairment of osteogenic differentiation of BMMSCs as compared with healthy controls ${ }^{27}$. In the current study, knockdown of Dicer1 in HC-MSCs decreased the osteogenic differentiation of MSCs. Moreover, MM-MSCs exhibited an increased tumor-supporting capacity. Thus, the senile MSCs could be a promoting element in favoring myeloma cell growth of patients with MM. Furthermore, knockdown of Dicer1 in HC-MSCs promoted cellular senescence and tumor-supporting capacities, while decreasing the differentiation of MSCs. We confirmed that MM-MSCs and Dicer1-KD MSCs increased the

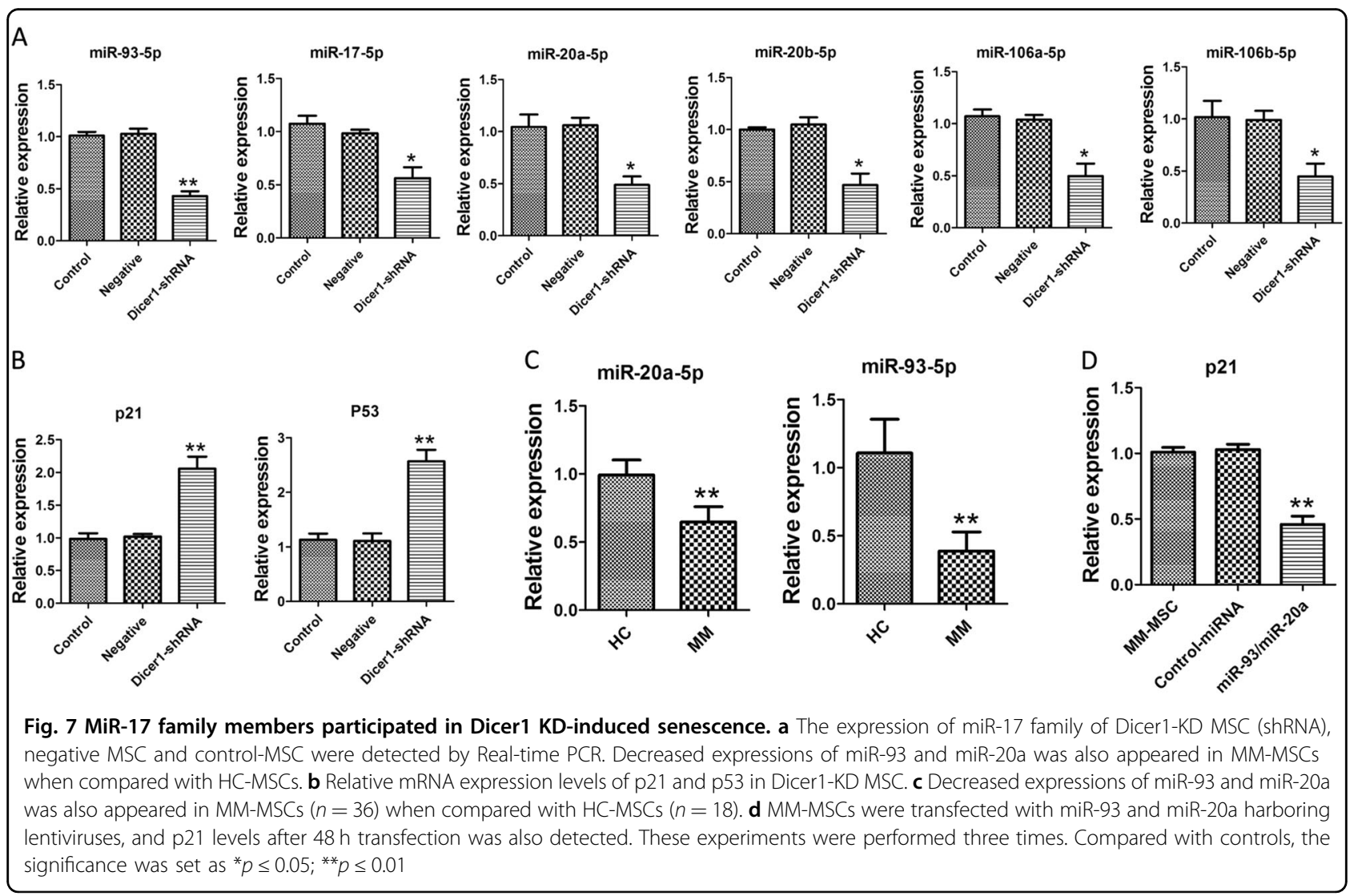


proportions of $\mathrm{S}$ phase myeloma cells. In accordance with our findings, some studies showed that MM-MSCs displayed tumor-supporting capability compared with healthy controls. However, some of the previous reports demonstrated that MSCs possibly have inhibition capability. For example, Ramasamy et al. reported that MSCs act directly on tumor cells, suppressing proliferation and inducing apoptosis in tumor cells ${ }^{28}$. Whether MSCs promote or inhibit myeloma cell growth remains controversial. The diversities in these findings are probably due to the different origin of MSCs, cellular populations and experimental protocols in the respective studies.

In recent years, miRNAs, a pivotal group of moderating factors, have been deemed to be prominent regulators of cellular senescence ${ }^{18,19}$. It is reported that Dicer1 is an RNAse III endonuclease responsible for microRNA biogenesis $^{22}$. Furthermore, our preliminary findings showed that the mRNA and protein levels of Dicer1 in MSCs isolated from MDS patients was significantly decreased compared with MSCs from health controls ${ }^{17}$. On the basis of these findings, we evaluated Dicer1 expression in MMMSCs. It was demonstrated that the expression of Dicer1 decreased in primary and expanded MM-MSCs. In the current study, we investigated the effects of a lower expression of Dicer1 in MM-MSCs by generating a stable knockdown model. Our study is mainly concerned with the biological function of Dicer1 in cell senescence, differentiation and tumor support of Dicer1-KD MSC expedited cellar senescence, which manifested as flattened cell morphology, increased SA- $\beta$-gal positive incidence and a decreased percentage of cells in $S$ phase. Experiments involving Dicer1 overexpression further verified these results. Upregulation of Dicer1 expression in MMMSC reversed cellular senescence and promoted differentiation. Under-expression of Dicer1 interferes with the biogenesis of miRNA, which is relevant to cellular senescence in endothelial cells. However, the role of miRNA in the senescence of BMMSCs remains ambiguous. Senescent cells generally exhibit increased expression of cell cycle inhibiting factor such as p53/p21, which could be adjusted by miRNA ${ }^{29-31}$. In our current study, decreased expression of the miR-17 family (miR-106a/b, miR-17-5p, miR-20a/b, and miR-93) was determined to be a critical factor responsible for elevated $p 21$ expression in Dicer1-KD MSC. Actually, miRNAs in the miR-17 family have been identified as regulators of cell cycle through targeting p21 in many other studies ${ }^{32-34}$. It was demonstrated that a lower of expression of the miR-17 family could give rise to the Dicer1-KD-induced cell senescence in MSCs in our present and previous studies. Lately, substantial miRNA expression was reported to be downregulated in MM-MSCs, demonstrating that reduced miRNA expression probably be associated with the pathogenic mechanism of MM. Our result showed that expression of miR-93 and miR-20a remarkably decreased in MM-MSCs. Moreover, overexpression of miR-93/ miR-20a reversed cellular senescence in MMMSCs through targeting p21. It was confirmed that miRNA showed a critical effect on increasing cell senescence of MM-MSCs.

Dicer1-KD MSCs displayed diminished osteogenic differentiation potential, characterized by the reduction in the expression of osteoblastic maker genes, calcium deposits and ALP activity, which was consistent with previous studies. Furthermore, upregulation of Dicer1 increased the impaired osteoblastic differentiation capability of MM-MSCs. Recently, many studies demonstrated that osteolytic bone lesions of MM patients resulted from the abnormal osteoblastic function in patients $^{6,35-37}$. Our study reported that the reduction of miRNA processing induced by Dicer1-KD was effective to immensely decrease the differentiation ability of MSCs, which indicated that exact level of mature miRNAs is important for MSC osteoblast differentiation. Most importantly, it provides a new perspective for the study of bone lesions in MM.

The senescent MSCs not only exhibited an impaired differentiation potential but also an enhancement in their support of tumor growth. So far, knowledge concerning the effect of MSC senescence in tumor growth support is restricted. In our results, in addition to the classic features of cell senescence, Dicer1-KD MSCs exhibited a significant increase in their capacity to support myeloma cells. Furthermore, BMMSCs derived from patients with MM were found to deliver the cytokine IL-6, which is known to promote tumor formation and progression. The tumor microenvironment-derived GDF15 was also able to significantly increase cell survival in primary MM cells.

\section{Conclusions}

In this study, we explored the feature and molecular mechanism of MSCs senescence in MM patients. Dicer1 plays a crucial role in the senescent process of MM-MSCs by regulating miRNA. Senescent MM-MSCs have a decreased differentiation potential and an increased tumor-supporting capacity. Therefore, much attention should be paid to the therapeutic exploitation of MSCs in bone lesion diseases and tumor progression.

\section{Methods \\ Patients}

A total of forty-six patients with newly diagnosed MM $(n=46)$ were enrolled between January 2015 and June 2017 in this study (Table 1). All experiments were approved by the Ethics Committee of Shanghai Jiao tong University Affiliated Sixth People's Hospital (Shanghai, 


\begin{tabular}{ll}
$\begin{array}{l}\text { Table } \mathbf{1} \text { Clinical characteristics of patients with multiple } \\
\text { myeloma }\end{array}$ & $\mathbf{N}=\mathbf{4 6}$ \\
\hline Characteristic & \\
\hline Sex & $25(54 \%)$ \\
$\quad$ Male, $n(\%)$ & $21(46 \%)$ \\
Female, $n$ (\%) & $65(38-85)$ \\
Median age, years (range) & \\
Immunoglobulin subtype, $n(\%)$ & $22(48 \%)$ \\
IgG & $10(22 \%)$ \\
IgA & \\
Light-chain & $6(13 \%)$ \\
K & $2(4 \%)$ \\
$\lambda$ & $6(13 \%)$ \\
Non-secretory & \\
International staging system, $n(\%)$ & $22(48 \%)$ \\
I & $14(30 \%)$ \\
III & $10(22 \%)$ \\
\hline
\end{tabular}

China) and written informed consent was obtained from all subjects. Patients were diagnosed according to the criteria recently defined by the International Myeloma Working Group ${ }^{38}$, and were staged according to the criteria of ISS $^{39}$. For the patients enrolled, they were all uniformly treated with bortezomib and dexamethasone. The median follow-up was 24 months (rang 9-38 months). The bone marrow was extracted for experiments before the treatment start. Eighteen healthy controls with the median age of 66 years (age range, 46-79 years) were analyzed in our study and they were matched for gender and age.

\section{Cell culture}

Bone marrow MSCs from patients and controls were isolated, cultured and harvested. The procedure was detailed in our previous studies. Human MM cell lines NCI-H929, OPM-2, and KMS-12-BM were cultured in RPMI 1640 medium (Gibco, Grand Island, NY, USA), supplemented with $10 \%$ fetal bovine serum (Gibco), 100 units $/ \mathrm{mL}$ penicillin, and $100 \mathrm{mg} / \mathrm{mL}$ streptomycin.

\section{Cell proliferation}

The proliferation of BMMSCs was detected by using CCK-8 (Dojindo, Japan) and by measuring the cell doubling time from P1 to P5. To evaluate the clonogenic potential of BMMSCs, colony-forming unit fibroblast (CFU-F) at P1, P3, and P5 were counted (colonies were stained with Giemsa).
Co-culture of BMMSCs with myeloma cells and 5-Ethynyl2'-deoxyuridine (EdU) incorporation assay

The human multiple myeloma cell lines NCI-H929 were purchased from the American Type Culture Collection (ATCC, Manassas, VA, USA). The cells were cultured in RPMI 1640 (Gibco) medium supplemented with 10\% fetal bovine serum (Gibco),100 units/mL penicillin, and 100 $\mathrm{mg} / \mathrm{mL}$ streptomycin in a humidified atmosphere with $5 \%$ $\mathrm{CO}_{2}$ at $37^{\circ} \mathrm{C}$. NCI-H929 were co-cultured with MSCs (HC-MSCs or MM-MSC) for $24 \mathrm{~h}$. We used a trans well inserts with $1-\mu \mathrm{m}$ pores. MM cell lines or MM primary cells were cultured in the upper chamber of the inserts. (For cell cycle and proliferation analysis, cells were exposed to $10 \mu \mathrm{M}$ EdU (Invitrogen) in culture medium for $45 \mathrm{~min}$. EdU was detected with Alexa Fluor 647-azide with the use of a Click-iT EdU Flow Cytometry Assay Kit (Invitrogen). Flow cytometry was performed with a FACS Calibur flow cytometer (Becton Dickinson, SanJose, CA, USA) and Cell Quest software.

\section{SA- $\beta$-gal assay}

BMMSCs were plated into 6 well culture-plates and maintained until $80 \%$ confluence. The cells were incubated with SA- $\beta$-gal staining solution (Beyotime, China) according to the manufacturer's instructions. Under inverted microscope, the number of SA- $\beta$-gal positive cells (blue cells) were calculated as senescent cells.

\section{Cell cycle analysis}

Cells were fixed by precooled ethanol for at least $24 \mathrm{~h}$ and stained with the PI/RNase Staining Solution kit (Invitrogen, Carlsbad, CA, USA) according to the manufacturer's directions. The cell cycle analysis was evaluated by flow cytometry and MultiCycle software.

\section{Cell apoptosis analysis}

Apoptosis of MM cells was induced by Bortezomib (Selleck, Shanghai, China). The quantification of apoptotic cells was performed by using the AnnexinV-FITC Apoptosis Detection Kit (Invitrogen, Carlsbad, CA, USA) according to the manufacturer's instructions. The analyses were performed on the FACScan flow cytometer.

\section{Real-time PCR}

Total RNA was isolated using the RNeasy Mini Kit (Qiagen, Germany) according to the manufacturer's instructions. For mRNA detection, reverse transcription of RNA was performed using the ReverTra Ace qPCR RT Kit (TOYOBO, Osaka, Japan), and real-time PCR (RTPCR) was carried out using SYBR ${ }^{\circledR}$ Premix Ex Taq ${ }^{\text {Ta }}$ II (Tli RNaseH Plus) (Takara, Kusatsu, Shiga, Japan). The primers are listed in Supplementary Table. The process was detailed in our previous study ${ }^{40}$. 


\section{Western blotting}

The cellular lysate was fractionated by $12 \%$ SDS-PAGE and electroblotted onto PVDF membranes. Membranes were incubated with primary antibodies including antiDicer1 (rabbit, ABclonal, USA), anti-p21 (rabbit, ABclonal, USA) and anti-ßactin (rabbit, ABclonal, USA). Secondary goat anti-rabbit antibodies labeled with horseradish peroxidase (Amersham Biosciences) were used. The process was detailed in our previous study ${ }^{17,41}$.

\section{Dicer1 shRNA transductions}

The HC-MSCs were transfected with shDicer1-eGFP vectors (shRNA group) and negative vector. And detailed information about cell transductions is provided in our previous articles ${ }^{17}$.

\section{Cell transductions of adenoviral vectors for expression of Dicer1}

The process of the construction of adenoviral vectors for expression of Dicer1 was detailed previously ${ }^{17},{ }^{41}$. The MM-MSCs were planted in 6-well plates and cultivated for $24 \mathrm{~h}$. The cells were transfected with $20 \mu \mathrm{l}$ adenoviral vectors and cultured for $12 \mathrm{~h}$ and then the medium was changed. The cells were cultured for another $36 \mathrm{~h}$ and harvested. The transfection was performed according to the manufacturer's protocol.

\section{MicroRNA-93/microRNA-20a overexpression}

MM-MSC were transfected with human miR-20a, miR93 and scrambled control lentivirus (Genechem Company, Shanghai, China) according to the manufacturer's protocol.

\section{Statistical analysis}

The data were presented as mean \pm SD. All statistical analyses were performed using the SPSS 17.0 System. Comparison of mRNA levels between healthy controls and different MM subtypes was using a Student's $t$ test, one-way analysis of variance (ANOVA) was used to assess multiple pairwise comparisons $p<0.05$ was considered statistically significant. Estimation of PFS was performed using the method of Kaplan and Meier.

\section{Acknowledgements}

This project was funded by the National Natural Science Foundation of China (Grant nos. 81570108, 81770121, and 81600095).

\section{Conflict of interest}

The authors declare that they have no conflict of interest.

\section{Publisher's note}

Springer Nature remains neutral with regard to jurisdictional claims in published maps and institutional affiliations.

Supplementary Information accompanies this paper at https://doi.org/ 10.1038/s41419-018-0545-6.
Received: 7 December 2017 Revised: 12 March 2018 Accepted: 27 March 2018

Published online: 03 May 2018

References

1. Palumbo, A. \& Anderson, K. Multiple myeloma. N. Engl. J. Med. 364, 1046-1060 (2011).

2. Bergfeld, S. A. \& DeClerck, Y. A. Bone marrow-derived mesenchymal stem cells and the tumor microenvironment. Cancer Metastas. Rev. 29, 249-261 (2010).

3. Arnulf, B. et al. Phenotypic and functional characterization of bone marrow mesenchymal stem cells derived from patients with multiple myeloma. Leukemia 21, 158-163 (2007).

4. Corre, J. et al. Bone marrow mesenchymal stem cells are abnormal in multiple myeloma. Leukemia 21, 1079-1088 (2007).

5. Reagan, M. R. \& Ghobrial, I. M. Multiple myeloma mesenchymal stem cells: characterization, origin, and tumor-promoting effects. Clin. Cancer Res. Off. J. Am. Assoc. Cancer Res. 18, 342-349 (2012).

6. Xu, S. et al. Impaired osteogenic differentiation of mesenchymal stem cells derived from multiple myeloma patients is associated with a blockade in the deactivation of the Notch signaling pathway. Leukemia 26, 2546-2549 (2012).

7. De Veirman, K. et al. Induction of miR-146a by multiple myeloma cells in mesenchymal stromal cells stimulates their pro-tumoral activity. Cancer Lett. 377, 17-24 (2016)

8. Bonab, M. M. et al. Aging of mesenchymal stem cell in vitro. BMC Cell. Biol. 7, 14 (2006).

9. Berenstein, R. et al. Multiple myeloma cells alter the senescence phenotype of bone marrow mesenchymal stromal cells under participation of the DLK1DIO3 genomic region. BMC Cancer 15, 68 (2015).

10. Stenderup, K., Justesen, J., Clausen, C. \& Kassem, M. Aging is associated with decreased maximal life span and accelerated senescence of bone marrow stromal cells. Bone 33, 919-926 (2003).

11. Zhou, S. et al. Age-related intrinsic changes in human bone-marrow-derived mesenchymal stem cells and their differentiation to osteoblasts. Aging Cell 7, 335-343 (2008).

12. Coppe, J. P., Desprez, P. Y., Krtolica, A. \& Campisi, J. The senescence-associated secretory phenotype: the dark side of tumor suppression. Annu. Rev. Pathol. 5, 99-118 (2010).

13. Campisi, J. \& d'Adda di Fagagna, F. Cellular senescence: when bad things happen to good cells. Nat. Rev. Mol. Cell. Biol. 8, 729-740 (2007).

14. van Deursen, J. M. The role of senescent cells in ageing. Nature $\mathbf{5 0 9}, \mathbf{4 3 9 - 4 4 6}$ (2014).

15. Andre, T. et al. Evidences of early senescence in multiple myeloma bone marrow mesenchymal stromal cells. PLoS ONE 8, e59756 (2013).

16. Fei, $C$. et al. Senescence of bone marrow mesenchymal stromal cells is accompanied by activation of p53/p21 pathway in myelodysplastic syndromes. Eur. J. Haematol. 93, 476-486 (2014).

17. Zhao, Y. et al. Down-regulation of Dicer1 promotes cellular senescence and decreases the differentiation and stem cell-supporting capacities of mesenchymal stromal cells in patients with myelodysplastic syndrome. Haematologica 100, 194-204 (2015).

18. Inukai, S. \& Slack, F. MicroRNAs and the genetic network in aging. J. Mol. Biol. 425, 3601-3608 (2013).

19. Martinez, l., Almstead, L. L. \& DiMaio, D. MicroRNAs and senescence. Aging 3, 77-78 (2011)

20. $\mathrm{Xu}$, S. et al. Upregulation of miR-135b is involved in the impaired osteogenic differentiation of mesenchymal stem cells derived from multiple myeloma patients. PLOS ONE 8, e79752 (2013).

21. Marasa, B. S. et al. MicroRNA profiling in human diploid fibroblasts uncovers miR-519 role in replicative senescence. Aging 2, 333-343 (2010).

22. Mudhasani, R. et al. Loss of miRNA biogenesis induces p19Arf-p53 signaling and senescence in primary cells. J. Cell. Biol. 181, 1055-1063 (2008).

23. Ungvari, Z. et al. Aging-induced dysregulation of dicer1-dependent microRNA expression impairs angiogenic capacity of rat cerebromicrovascular endothelial cells. J. Gerontol. A. Biol. Sci. Med. Sci. 68, 877-891 (2013).

24. Iliou, M. S. et al. Impaired DICER1 function promotes stemness and metastasis in colon cancer. Oncogene 33, 4003-4015 (2014).

25. Yaccoby, S. et al. Inhibitory effects of osteoblasts and increased bone formation on myeloma in novel culture systems and a myelomatous mouse model. Haematologica 91, 192-199 (2006). 
26. Bisping, $G$. et al. Paracrine interactions of basic fibroblast growth factor and interleukin-6 in multiple myeloma. Blood 101, 2775-2783 (2003).

27. Guo, J. et al. Lenalidomide restores the osteogenic differentiation of bone marrow mesenchymal stem cells from multiple myeloma patients via deactivating Notch signaling pathway. Oncotarget 8, 55405-55421 (2017).

28. Ramasamy, R. et al. Mesenchymal stem cells inhibit proliferation and apoptosis of tumor cells: impact on in vivo tumor growth. Leukemia 21, 304-310 (2007).

29. Ksiazek, K. A comprehensive review on mesenchymal stem cell growth and senescence. Rejuvenation. Res. 12, 105-116 (2009).

30. Ivanovska, I. et al. MicroRNAs in the miR-106b family regulate p21/CDKN1A and promote cell cycle progression. Mol. Cell. Biol. 28, 2167-2174 (2008).

31. Andl, $T$. et al. The miRNA-processing enzyme dicer is essential for the morphogenesis and maintenance of hair follicles. Curr. Biol. CB 16, 1041-1049 (2006).

32. Gibcus, J. H. et al. MiR-17/106b seed family regulates p21 in Hodgkin's lymphoma. J. Pathol. 225, 609-617 (2011).

33. Wang, Z. et al. Suppression of p21 by c-Myc through members of miR-17 family at the post-transcriptional level. Int. J. Oncol. 37, 1315-1321 (2010).

34. Kim, Y. K. et al. Functional links between clustered microRNAs: suppression of cell-cycle inhibitors by microRNA clusters in gastric cancer. Nucl. Acids Res. 37, 1672-1681 (2009)
35. El Khassawna, T. et al. Impaired extracellular matrix structure resulting from malnutrition in ovariectomized mature rats. Histochem. Cell. Biol. 144, 491-507 (2015).

36. Silvestris, F., Cafforio, P., Calvani, N. \& Dammacco, F. Impaired osteoblastogenesis in myeloma bone disease: role of upregulated apoptosis by cytokines and malignant plasma cells. Br. J. Haematol. 126, 475-486 (2004).

37. Yaccoby, S. Osteoblastogenesis and tumor growth in myeloma. Leuk. Lymphoma 51, 213-220 (2010).

38. International Myeloma Working Group. Criteria for the classification of monoclonal gammopathies, multiple myeloma and related disorders: a report of the International Myeloma Working Group. Br. J. Haematol. 121, 749-757 (2003).

39. Durie, B. G. \& Salmon, S. E. A clinical staging system for multiple myeloma. Correlation of measured myeloma cell mass with presenting clinical features, response to treatment, and survival. Cancer 36, 842-854 (1975).

40. Fei, C. et al. Impaired osteogenic differentiation of mesenchymal stem cells derived from bone marrow of patients with lower-risk myelodysplastic syndromes. Tumour Biol. J. Int. Soc. Oncodev. Biol. Med. 35, 4307-4316 (2014).

41. Fei, $\mathrm{C}$. et al. Notch-Hes pathway mediates the impaired osteogenic differentiation of bone marrow mesenchymal stromal cells from myelodysplastic syndromes patients through the down-regulation of Runx2. Am. J. Transl. Res. 7, 1939-1951 (2015) 